\title{
Dynamic synthesis of machine with slider-crank mechanism
}

\author{
A. A. Jomartov ${ }^{1}$, S. U. Joldasbekov ${ }^{1}$, and Yu. M. Drakunov ${ }^{2}$ \\ ${ }^{1}$ Institute Mechanics and Mechanical Engineering, Almaty, Kazakhstan \\ 2 al-Farabi Kazakh National University, Almaty, Kazakhstan \\ Correspondence to: A. A. Jomartov (legsert@mail.ru)
}

Received: 1 November 2014 - Revised: 12 March 2015 - Accepted: 21 March 2015 - Published: 2 April 2015

\begin{abstract}
In this paper we consider the formulation and solution of the task of a dynamic synthesis machine with an asynchronous electric motor and a slider-crank mechanism. The constant parameters of the slider-crank mechanism (mass and moments of inertia and centers of gravity of links) and the parameters of the electrical motor are defined. The laws of motion of the machine and kinematic parameters of the mechanism are considered as given. We have developed the method of optimal dynamic synthesis of the machine, which consists of an asynchronous electric motor and a slider-crank mechanism. The criterion of optimization of the dynamic synthesis of a machine is the root mean square sum of the moments of driving forces, the forces of resistance and inertia forces which are reduced to the axis of rotation of the crank. The method of optimal dynamic synthesis of a machine can be used in the design of new and the improvement of known mechanisms and machines.
\end{abstract}

\section{Introduction}

The dynamic synthesis of mechanisms is among the most important and difficult problems encountered in the design of machines (Dresig and Vulfson, 1990; Dresig and Holzweißig, 2010; Wittenbauer, 1923; Homer and Eckhardt, 1998; Browne, 1965; Helmi and Hassan, 2008; Shabana, 1998; Erdman and Sandor, 1984). The main task of the dynamic synthesis of mechanisms is to determine the optimal values of the parameters and their combinations. The simplest task of the dynamic synthesis in the field of mechanics of machines is the Wittenbauer method (Wittenbauer, 1923) for determining the moment of inertia of the flywheel and the constant drive torque on the motor shaft. This method is a graph-analytical method and is based on plotting the energy-mass. Kolovsky et al. (2000) shows the method of dynamic analysis and synthesis of the machine. Shchepetilnikov (1975) has researched the static and dynamic balancing of different mechanisms. The solution of problems in the dynamics synthesis of machines depends significantly on the correct representation of the allowable levels of dynamic errors and dynamic forces. In many cases it is possible to allow significant dynamic errors in systems by introducing the necessary adjustment units during design. This is particularly obvious for cyclic machines working under steady state conditions; in such machines dynamic errors in the equations of motion of the working components can be compensated by adjustment mechanisms within defined limits (Astashev, Babitsky, and Kolovsky, 2000). The general tasks of dynamic synthesis have been set by various criteria: the minimization of reactions in the bearings, minimum deviation of the angular acceleration of the driving link, etc. (Penun uri, et al., 2011; Guangping and Zhiyong, 2011; Volkert and Herder, 2012; Wunderlich, 1968; Kim and Hee, 2012). While solving the dynamic problems, the analysis and synthesis are usually closely connected. In particular, many of the problems of synthesis, which establish the rational values of the system's parameters, often base on preliminarily solved problem of analysis. One of the most important and urgent tasks, for consideration, is the development of the optimization criteria. These criteria should be based on the most significant factors of the considered problem and at the same time have the foreseeable shape, to retain the role of active tool for the dynamics synthesis, when developing variants of a machine. When taking into account the elasticity of links, the question of criteria, without losing its importance, is further complicated. In this case, in addition to geometric and kinematic character- 


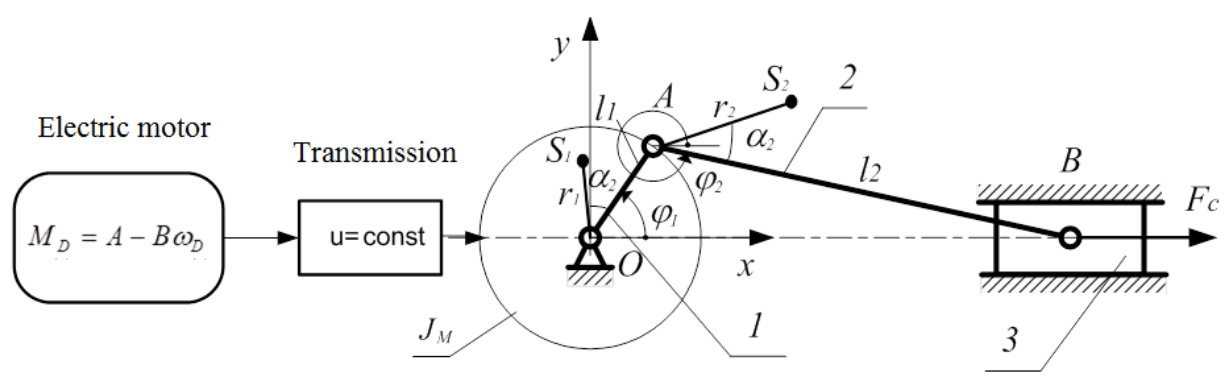

Figure 1. Diagram of the machine.

istics, other factors, characterizing the frequency features of the system, level of proximity of working models to the dynamically unstable modes, level of additional dynamic load, caused by oscillations and many other factors appear as dynamic criteria (Vulfson, 2015). The aim of this paper is to develop the method of optimal dynamic synthesis of the machine, which consists of a motor and a mechanism, using the methods of synthesis and choosing the optimization criteria which were proposed in the work (Vulfson, 2015).

\section{Dynamic synthesis of a machine}

Problem statement. Machine motion with rigid links is described by the following equations:

$J_{n} \ddot{\varphi}_{1}+\frac{1}{2} \frac{d J_{n}}{d \varphi_{1}} \dot{\varphi}_{1}^{2}=Q=M_{D}+M_{C}\left(\varphi_{1}, \dot{\varphi}_{1}\right)$

$\tau \dot{M}_{D}+M_{D}=A-B \dot{\varphi}_{1}$, at $\tau \cong 0, M_{D}=A-B \dot{\varphi}_{1}$.

Where $\varphi_{1}$-generalized coordinate, $J_{n}$ - the reduced moment of inertia, $Q$ - the generalized force, $M_{D}$ - the torque of asynchronous electric motor, $M_{C}\left(\varphi_{1}, \dot{\varphi}_{1}\right)$ - the resistance moment, $A, B$ - the parameters of the static characteristics of asynchronous electric motor, $\tau$ - the electrical time constant of the asynchronous electric motor.

Let's solve the problem of dynamic synthesis of machine, which is to determine the parameters of its mechanisms (mass and moments of inertia and the centre of gravity of links) and motor parameters, the law of motion of the machine set $\phi_{1}^{*}=\phi_{1}^{*}(t)$ or $\omega_{1}^{*}=\omega_{1}^{*}(t)$ and the kinematic parameters of the mechanism are considered as given. As the objective function for the dynamic synthesis of machine, we construct a functional on the basis of Eq. (1), with taking into account the characteristics of the motor (Eq. 2).

$G=\sum_{i=1}^{n}\left[J_{n} \ddot{\varphi}_{1 i}+\frac{1}{2} \frac{\partial J_{n}}{\partial \varphi_{1}} \dot{\varphi}_{1 i}^{2}-Q_{i}\right]^{2}$.

The required parameters of the machine are determined by minimizing the root mean square sum of the moments of driving forces, the forces of resistance and inertia forces which are reduced to the axis of rotation of the crank, for the $n$ positions of the mechanism.
Let us consider the optimal dynamic synthesis of a machine, which consists of an asynchronous electric motor and a slider-crank mechanism. As a criterion for the optimization of the dynamic synthesis machine we choose the root mean square sum of the moments of driving forces, the forces of resistance and inertia forces which are reduced to the axis of rotation of the crank, for the $n$ positions of the slider-crank mechanism. A diagram of the machine is shown in Fig. 1.

By solving the task of kinematic analysis of the slidercrank mechanism (see. Fig. 1), taking into account, we have the formulas

$\left\{\begin{array}{l}\varphi_{2}=2 \pi-\arcsin \left(\lambda \sin \varphi_{1}\right), \\ x_{C}=l_{1} \cos \varphi_{1}+l_{2} \cos \varphi_{2} \\ x_{S_{2}}=l_{1} \cos \varphi_{1}+r_{2} \cos \left(\varphi_{2}+\alpha_{2}\right), \\ y_{S_{2}}=l_{1} \sin \varphi_{1}+r_{2} \sin \left(\varphi_{2}+\alpha_{2}\right)\end{array}\right.$

After differentiation of Eq. (4) by the generalized coordinates, we obtain the first transfer functions and second transfer functions

$$
\left\{\begin{array}{l}
\varphi^{\prime}{ }_{2}=-\lambda \frac{\cos \varphi_{1}}{\cos \varphi_{2}}, \\
x^{\prime}{ }_{C}=l_{1} \sin \varphi_{1}\left(\varphi^{\prime}-1\right) \\
x^{\prime}{ }_{S_{2}}=-l_{1} \sin \varphi_{1}-r_{2} \sin \left(\varphi_{2}+\alpha_{2}\right) \cdot \varphi^{\prime}, \\
y^{\prime} S_{2}=l_{1} \cos \varphi_{1}+r_{2} \cos \left(\varphi_{2}+\alpha_{2}\right) \cdot \varphi^{\prime}{ }_{2} \\
\varphi^{\prime \prime}{ }_{2}=\lambda \frac{\sin \left(\varphi_{1}-\varphi_{2}\right)}{\cos ^{2} \varphi_{2}}, \\
x^{\prime \prime}{ }_{C}=l_{1}\left[\cos \varphi_{1}\left(\varphi_{2}^{\prime}-1\right)+\sin \varphi_{1} \cdot \varphi^{\prime \prime}{ }_{2}\right] \\
x^{\prime \prime}{ }_{S_{2}}=-l_{1} \cos \varphi_{1}-r_{2}\left[\cos \left(\varphi_{2}+\alpha_{2}\right) \cdot \varphi_{2}^{\prime 2}\right. \\
y^{\prime \prime}{ }_{S_{2}}=-l_{1} \sin \varphi_{1}-r_{2}\left[\sin \left(\varphi_{2}+\alpha_{2}\right) \cdot \varphi_{2}^{\prime 2}\right. \\
\left.\quad+\sin \left(\varphi_{2}+\alpha_{2}\right) \cdot \varphi^{\prime \prime}{ }_{2}\right], \\
\left.\quad-\cos \left(\varphi_{2}+\alpha_{2}\right) \cdot \varphi^{\prime \prime}{ }_{2}\right]
\end{array}\right.
$$

The input parameters of the dynamic synthesis of a machine are: $F_{C}$ - the force of technological resistance; $\phi_{1}, \dot{\phi}_{1}, \ddot{\phi}_{1}$ the generalized coordinates and the velocities and accelerations of the crank $1 ; l_{1}$ and $l_{2}$ - the geometric dimensions of the coupler and crank; $u=\omega_{D} / \omega_{1}-$ the transmission ratio; $\omega_{\mathrm{c}}-$ the average angular velocity of the crank in a steady motion, and $\delta$ - the coefficient of unevenness of movement.

The output parameters of dynamic synthesis are: $m_{1}, m_{2}$, $m_{3}$ - the mass of the crank, coupler and slider; $a_{1}=r_{1} \cos \alpha_{1}$, $b_{1}=r_{1} \sin \alpha_{1}, a_{2}=r_{2} \cos \alpha_{2}, b_{2}=r_{2} \sin \alpha_{2}-$ the coordinates 


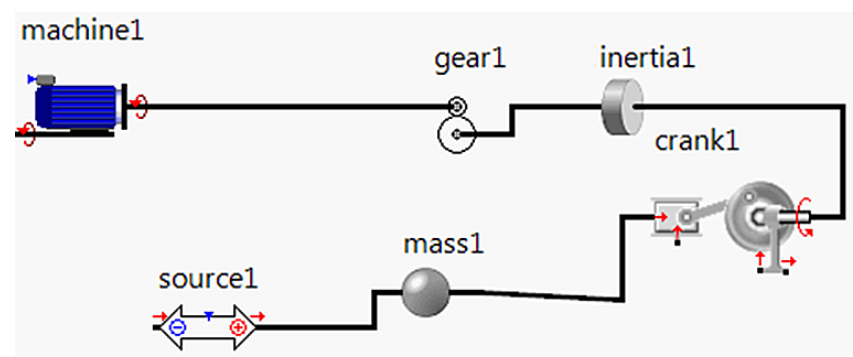

Figure 2. Dynamic model of machine with slider-crank mechanism on SimulationX.

of the centers of mass of the crank and coupler; $J_{D}-$ the moment of inertia of the rotor of the electric motor; $J_{M}$ - the moment of inertia of the flywheel; $J_{1}, J_{2}$ - the moments of inertia of the crank and coupler.

The parameters of the machine are determined from the condition of the minimum root mean square sum of the moments of driving forces, the forces of resistance and inertia forces which are reduced to the axis of rotation of the crank, for the $n$ positions of the slider-crank mechanism.

This optimization criterion is given by

$G=\sum_{i=1}^{n}\left[J_{n} \ddot{\varphi}_{1 i}+\frac{1}{2} \frac{\partial J_{n}}{\partial \varphi_{1}} \dot{\varphi}_{1 i}^{2}-Q_{i}\right]^{2}$

In Eq. (6) the reduced moment of inertia of the machine is given by

$J_{n}=J_{D} u^{2}+J_{M}+J_{1}+m_{3} x_{C}^{\prime 2}+m_{2}\left(x_{S_{2}}^{\prime 2}+y_{S_{2}}^{\prime 2}\right)+J_{2} \varphi_{2}^{\prime 2}$

and the remaining parameters are determined by the expressions:

$$
\begin{aligned}
& \frac{1}{2} \frac{\partial J_{n}}{\partial \phi_{1}}=m_{3} x^{\prime}{ }_{C} x^{\prime \prime}{ }_{C}+m_{2}\left(x^{\prime}{ }_{S_{2}} x^{\prime \prime} S_{2}+y^{\prime}{ }_{S_{2}} y^{\prime \prime}{ }_{S_{2}}\right)+J_{2} \phi_{2}^{\prime}{ }_{2}{ }^{\prime \prime}{ }_{2} \\
& Q=M_{D} u+F_{C} x^{\prime}{ }_{C}-m_{1} g\left(a_{1} \cos \varphi_{1}-b_{1} \sin \varphi_{1}\right)-m_{2} g y_{S_{2}}^{\prime}
\end{aligned}
$$

Let us write the expression $M_{D}$ through the parameters $A$ and $B$ the static characteristics of the asynchronous electric motor.

Then

$$
\begin{aligned}
& Q=\left(A-B u \dot{\phi}_{1}\right) u+F_{C} x_{C}^{\prime}-m_{1} g\left(a_{1} \cos \phi_{1}\right. \\
& \left.-b_{1} \sin \phi_{1}\right)-m_{2} g y^{\prime}{ }_{S_{2}} .
\end{aligned}
$$

We substitute the expressions of first transfer functions (Eq. 5) in Eq. (7)

$$
\begin{aligned}
& J_{n}=J_{0}+m_{3} l_{1}^{2} \sin ^{2} \phi_{1}\left(\phi_{2}^{\prime}-1\right)^{2}+J_{B} \phi_{2}^{\prime 2}+ \\
& 2 m_{2} l_{1} a_{2} \cos \left(\phi_{2}-\phi_{1}\right) \phi_{2}^{\prime}-2 m_{2} l_{1} b_{2} \sin \left(\phi_{2}-\phi_{1}\right) \phi_{2}^{\prime} .
\end{aligned}
$$

In Eq. (8) we introduce the notation

$$
J_{0}=J_{D} u^{2}+J_{M}+J_{1}+m_{2} l_{1}^{2}, J_{B}=J_{2}+m_{2}\left(a_{2}^{2}+b_{2}^{2}\right) .
$$

The partial derivative of the reduced moment of inertia is

$$
\begin{aligned}
& \frac{1}{2} \frac{\partial J_{n}}{\partial \phi_{1}}=m_{3} l_{1}^{2} \sin \phi_{1}\left[\cos \phi_{1}\left(\phi_{2}^{\prime}-1\right)+\sin \phi_{1} \phi_{2}^{\prime \prime}\right]\left(\phi_{2}^{\prime}-1\right) \\
& +J_{B} \phi_{2}^{\prime} \phi_{2}^{\prime \prime}{ }_{2}++m_{2} l_{1} a_{2}\left[\cos \left(\phi_{2}-\phi_{1}\right) \phi_{2}^{\prime \prime}\right. \\
& \left.-\sin \left(\phi_{2}-\phi_{1}\right)\left(\phi_{2}^{\prime}-1\right) \phi_{2}^{\prime}\right]-m_{2} l_{1} b_{2} \\
& {\left[\sin \left(\phi_{2}-\phi_{1}\right) \phi_{2}^{\prime \prime}+\cos \left(\phi_{2}-\phi_{1}\right)\left(\phi_{2}^{\prime}-1\right) \phi_{2}^{\prime}\right] .}
\end{aligned}
$$

The final expression for the generalized force can be written $Q=\left(A-B u \dot{\phi}_{1}\right) u+F_{C} l_{1} \sin \phi_{1}\left(\phi_{2}^{\prime}-1\right)-m_{1} g a_{1} \cos \phi_{1}$ $+m_{1} g b_{1} \sin \phi_{1}-m_{2} g l_{1} \cos \phi_{1}-m_{2} g a_{2} \cos \phi_{2} \cdot \phi_{2}^{\prime}$

$+m_{2} g b_{2} \sin \phi_{2} \cdot \phi_{2}^{\prime}$.

The expression enclosed in square brackets of Eq. (6) is called a function of deviation $\Delta$. It is based on the relations (Eqs. 8-10) and is the form

$$
\begin{aligned}
& \Delta=J_{0} \ddot{\phi}_{1}+m_{3} l_{1}^{2} \sin ^{2} \phi_{1}\left(\phi_{2}^{\prime}-1\right)^{2} \ddot{\phi}_{1}+J_{B} \phi_{2}^{\prime 2} \ddot{\phi}_{1} \\
& +2 m_{2} l_{1} a_{2} \cos \left(\phi_{2}-\phi_{1}\right) \phi_{2}^{\prime} \ddot{\phi}_{1}- \\
& -2 m_{2} l_{1} b_{2} \sin \left(\phi_{2}-\phi_{1}\right) \phi_{2}^{\prime} \ddot{\phi}_{1} \\
& +m_{3} l_{1}^{2} \sin \phi_{1}\left[\cos \phi_{1}\left(\phi_{2}^{\prime}{ }_{2}-1\right)+\sin \phi_{1} \phi_{2}^{\prime \prime}{ }_{2}\right]\left(\phi_{2}^{\prime}-1\right) \dot{\phi}_{1}^{2} \\
& +J_{B} \phi_{2}^{\prime} \phi^{\prime \prime}{ }_{2} \dot{\phi}_{1}^{2}+m_{2} l_{1} a_{2} \\
& {\left[\cos \left(\phi_{2}-\phi_{1}\right) \phi^{\prime \prime}{ }_{2}-\sin \left(\phi_{2}-\phi_{1}\right)\left(\phi_{2}^{\prime}-1\right) \phi_{2}^{\prime}{ }_{2}\right] \dot{\phi}_{1}^{2}-} \\
& m_{2} l_{1} b_{2}\left[\sin \left(\phi_{2}-\phi_{1}\right) \phi_{2}^{\prime \prime}{ }_{2}+\cos \left(\phi_{2}-\phi_{1}\right)\left(\phi_{2}^{\prime}-1\right) \phi_{2}^{\prime}{ }_{2}\right] \dot{\phi}_{1}^{2} \\
& -\left(A-B u \dot{\phi}_{1}\right) u-F_{C} l_{1} \sin \phi_{1}\left(\phi_{2}^{\prime}-1\right) \\
& +m_{1} g a_{1} \cos \phi_{1}-m_{1} g b_{1} \sin \phi_{1}+m_{2} g l_{1} \cos \phi_{1}+ \\
& m_{2} g a_{2} \cos \phi_{2} \cdot \phi_{2}^{\prime}-m_{2} g b_{2} \sin \phi_{2} \cdot \phi_{2}^{\prime} .
\end{aligned}
$$

Let us simplify and group the expression Eq. (11)

$$
\begin{aligned}
& \Delta=J_{0} \ddot{\phi}_{1}+m_{3} l_{1}^{2}\left\{\sin ^{2} \phi_{1}\left(\phi_{2}^{\prime}-1\right)^{2} \ddot{\phi}_{1}\right. \\
& \left.+\left[\sin 2 \phi_{1}\left(\phi_{2}^{\prime}-1\right) / 2+\sin ^{2} \phi_{1} \phi_{2}^{\prime \prime}{ }_{2}\right]\left(\phi_{2}^{\prime}-1\right) \dot{\phi}_{1}^{2}\right\}+ \\
& B u^{2} \dot{\phi}_{1}+m_{2} a_{2}\left\{2 l_{1} \cos \left(\phi_{2}-\phi_{1}\right) \phi_{2}^{\prime} \ddot{\phi}_{1}\right. \\
& +l_{1}\left[\cos \left(\phi_{2}-\phi_{1}\right) \phi_{2}^{\prime \prime}-\sin \left(\phi_{2}-\phi_{1}\right)\left(\phi_{2}^{\prime}-1\right) \phi_{2}^{\prime}\right] \dot{\phi}_{1}^{2} \\
& \left.+g \cos \phi_{2} \cdot \phi_{2}^{\prime}\right\}-m_{2} b_{2}\left\{2 l_{1} \sin \left(\phi_{2}-\phi_{1}\right) \phi_{2}^{\prime}\right. \\
& \ddot{\phi}_{1}+l_{1}\left[\sin \left(\phi_{2}-\phi_{1}\right) \phi^{\prime \prime}{ }_{2}+\cos \left(\phi_{2}-\phi_{1}\right)\right. \\
& \left.\left.\left(\phi_{2}^{\prime}-1\right) \phi_{2}^{\prime}\right] \dot{\phi}_{1}^{2}+g \sin \phi_{2} \cdot \phi_{2}^{\prime}\right\}+\left(m_{1} a_{1}\right. \\
& \left.+m_{2} l_{1}\right) g \cos \phi_{1}-m_{1} g b_{1} \sin \phi_{1}+J_{B}\left(\phi_{2}^{\prime 2} \ddot{\phi}_{1}+\right. \\
& \left.\phi_{2}^{\prime} \phi_{2}^{\prime \prime}{ }_{2} \dot{\phi}_{1}^{2}\right)-A u-F_{C} l_{1} \sin \phi_{1}\left(\phi_{2}^{\prime}-1\right) .
\end{aligned}
$$




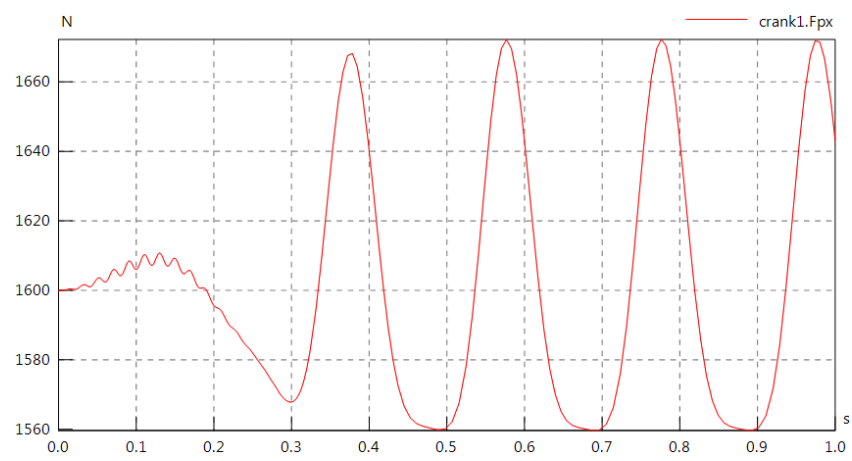

Figure 3. A graph of the longitudinal force acting on the slider for a typical machine.

The function of deviation $\Delta$ is represented as a generalized polynomial

$\Delta_{i}=\left[P_{1} f_{1}\left(\phi_{1 i}\right)+P_{2} f_{2}\left(\phi_{1 i}\right)+\ldots+P_{9} f_{9}\left(\phi_{1 i}\right)-F\left(\phi_{1 i}\right)\right]^{2}$.

Then the optimality criterion (Eq. 6) of the machine takes the form

$G=\sum_{i=1}^{n}\left[P_{1} f_{1}\left(\varphi_{1 i}\right)+P_{2} f_{2}\left(\varphi_{1 i}\right)+\ldots+P_{9} f_{9}\left(\varphi_{1 i}\right)-F\left(\varphi_{1 i}\right)\right]^{2}$.

Here, the following notation

$$
P_{1}=J_{0}, P_{2}=m_{1} b_{1}, P_{3}=J_{B}, P_{4}=m_{2} a_{2}, P_{5}=m_{2} b_{2},
$$$$
P_{6}=A u, P_{7}=B u^{2}, P_{8}=m_{1} a_{1}+m_{2} l_{1}, P_{9}=m_{3} l_{1}^{2} \text {. }
$$

$$
\begin{aligned}
& F\left(\varphi_{1}\right)=F_{C} l_{1} \sin \varphi_{1}\left(\varphi_{2}^{\prime}-1\right) \\
& f_{1}\left(\varphi_{1}\right)=\ddot{\varphi}_{1}, \\
& f_{2}\left(\varphi_{1}\right)=-g \sin \varphi_{1}, \\
& f_{3}\left(\varphi_{1}\right)=\varphi_{2}^{\prime 2} \ddot{\varphi}_{1}+\varphi_{2}^{\prime} \varphi_{2}^{\prime \prime}{ }_{2} \dot{\varphi}_{1}^{2}, \\
& f_{4}\left(\varphi_{1}\right)=2 l_{1} \cos \left(\varphi_{2}-\varphi_{1}\right) \varphi_{2}^{\prime} \ddot{\varphi}_{1}+l_{1}\left[\cos \left(\varphi_{2}-\varphi_{1}\right) \varphi_{2}^{\prime \prime}-\right. \\
& -\sin \left(\varphi_{2}-\varphi_{1}\right)\left(\varphi_{2}^{\prime}-1\right) \varphi_{2}^{\prime} \dot{\varphi}_{1}^{2}+g \cos \varphi_{2} \cdot \varphi_{2}^{\prime}, \\
& f_{5}\left(\varphi_{1}\right)=-2 l_{1} \sin \left(\varphi_{2}-\varphi_{1}\right) \varphi_{2}^{\prime} \ddot{\varphi}_{1}+ \\
& +l_{1}\left[\sin \left(\varphi_{2}-\varphi_{1}\right) \varphi_{2}^{\prime \prime}+\cos \left(\varphi_{2}-\varphi_{1}\right)\left(\varphi_{2}^{\prime}-1\right) \varphi_{2}^{\prime}\right] \dot{\varphi}_{1}^{2} \\
& +g \sin \varphi_{2} \cdot \varphi_{2}^{\prime}, \\
& f_{6}\left(\varphi_{1}\right)=-1, \\
& f_{7}\left(\varphi_{1}\right)=\dot{\varphi}_{1}, \\
& f_{8}\left(\varphi_{1}\right)=g \cos \varphi_{1}, \\
& f_{9}\left(\varphi_{1}\right)=\sin ^{2} \varphi_{1}\left(\varphi_{2}^{\prime}-1\right)^{2} \ddot{\varphi}_{1}+\left[\sin 2 \varphi_{1}\left(\varphi_{2}^{\prime}-1\right) / 2\right. \\
& \left.+\sin ^{2} \varphi_{1} \varphi_{2}^{\prime \prime}{ }_{2}\right]\left(\varphi_{2}^{\prime}-1\right) \dot{\varphi}_{1}^{2} .
\end{aligned}
$$

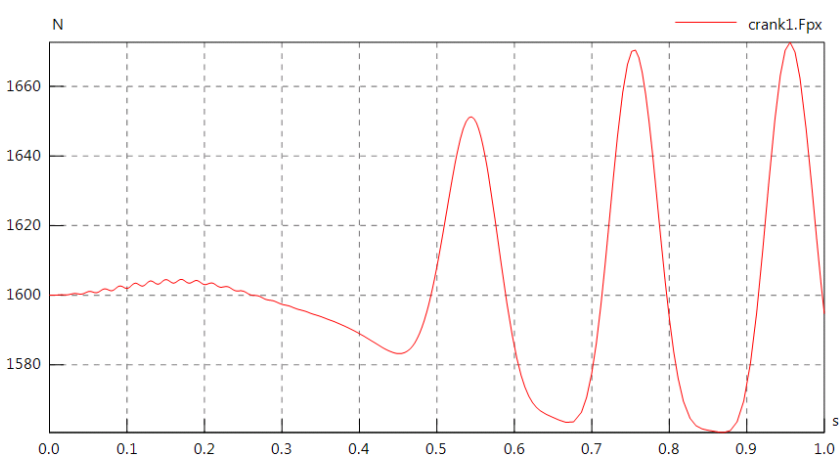

Figure 4. A graph of the longitudinal force acting on the slider for the synthesized machine with a slider-crank mechanism.

From the minimum of Eq. (12), we obtain a system of equations from which coefficients $P_{1}, P_{2}, \ldots, P_{9}$ are determined

$$
\frac{\partial G}{\partial P_{k}}=0, \quad k=1,2, \ldots, 9
$$

This system of equations in expanded form is:

$$
\left\{\begin{array}{l}
C_{11} P_{1}+C_{12} P_{2}+\ldots+C_{19} P_{9}=\sigma_{1} \\
C_{21} P_{1}+C_{22} P_{2}+\ldots+C_{29} P_{9}=\sigma_{2} \\
\ldots \ldots \ldots \ldots \ldots \ldots \ldots \ldots \ldots \ldots \ldots \\
C_{91} P_{1}+C_{92} P_{2}+\ldots+C_{99} P_{9}=\sigma_{9}
\end{array}\right.
$$

where

$$
\begin{aligned}
& C_{j k}=C_{k j}=\sum_{i=1}^{n} f_{j}\left(\phi_{1 i}\right) f_{k}\left(\phi_{1 i}\right), \\
& \sigma_{k}=\sum_{i=1}^{n} F\left(\phi_{1 i}\right) f_{k}\left(\phi_{1 i}\right), \quad j, k=1,2, \ldots, 9
\end{aligned}
$$

After finding the coefficients $P_{1}, P_{2}, \ldots, P_{9}$, we define the physical parameters of the relations (Eq. 13).

An algorithm for solving the above task is as follows:

1. The segment $\phi_{1} \in[0,2 \pi]$ is split into $n$ equal parts.

2. Let us take as a first approximation for the angular velocity of the following law.

$\omega_{1}=\dot{\varphi}_{1}=\omega_{\mathrm{c}}+0.5 \delta \omega_{\mathrm{c}} \cos \left(\varphi_{1}\right)$.

3. Then, the crank angular acceleration is determined by the formula

$$
\varepsilon_{1}=\ddot{\varphi}_{1}=-0.5 \delta \omega_{\mathrm{c}} \sin \left(\varphi_{1}\right) \cdot \dot{\varphi}_{1} .
$$

4. The coefficients $C_{j k}, \sigma_{k}$ are defined by Eq. (15);

5. We solve the system of linear equations (Eq. 14) and define the parameters $P_{k}$. 
The required physical parameters are determined from Eq. (13)

Typically, the mass $m_{3}$ of the slider is set on the basis of technological requirements. In this case, the order of the system of linear equations is reduced by 1 , i.e. $k=1,2, \ldots, 8$ and the final system becomes

$\sum_{j=1}^{8} C_{k j} P_{j}=\sigma_{k} \quad k=1,2, \ldots, 8$,

where

$C_{j k}=C_{k j}=\sum_{i=1}^{n} f_{j}\left(\phi_{1 i}\right) f_{k}\left(\phi_{1 i}\right), \sigma_{k}=\sum_{i=1}^{n} F\left(\phi_{1 i}\right) f_{k}\left(\phi_{1 i}\right)$,

$j, k=1,2, \ldots, 8$

Expression for the function $F\left(\phi_{1}\right)$ will be in the form

$F\left(\phi_{1}\right)=F_{C} l_{1} \sin \phi_{1}\left(\phi_{2}^{\prime}-1\right)-m_{3} l_{1}^{2}\left\{\sin ^{2} \phi_{1}\left(\phi_{2}^{\prime}-1\right)^{2} \ddot{\phi}_{1}+\right.$

$\left.\left[\sin 2 \phi_{1}\left(\phi_{2}^{\prime}-1\right) / 2+\sin ^{2} \phi_{1} \phi_{2}^{\prime \prime}{ }_{2}\right]\left(\phi_{2}^{\prime}-1\right) \dot{\phi}_{1}^{2}\right\}$.

The formation of the coefficients $C_{j k}, \sigma_{k}$ and the solution of () were carried out in the computing software Maple.

\section{Example}

Synthesis of the machine was carried out for the following initial data:

$m_{3}=1.4 \mathrm{~kg}, l_{1}=0.04 \mathrm{~m}, l_{2}=0.16 \mathrm{~m}, F_{C}=160 \mathrm{~N}$,

$u=5, \omega_{\mathrm{c}}=20 \mathrm{~s}^{-1}$.

The coefficients of the polynomial (Eq. 10), are: $P_{1}=$ $2.1022, \quad P_{2}=1.2619, \quad P_{3}=0.02478, \quad P_{4}=0.1263, \quad P_{5}=$ $0.2023, P_{6}=1522.8, P_{7}=50.627, P_{8}=8.1963$, The physical parameters of the machine are: $J_{0}=2.1022 \mathrm{~kg} \mathrm{~m}^{2}$, $J_{B}=0.02478 \mathrm{~kg} \mathrm{~m}^{2}, m_{1}=3.1263 \mathrm{~kg}, m_{2}=2.2023 \mathrm{~kg}, A=$ $304.56 \mathrm{Nm}, B=2.015 \mathrm{Nms}$.

The angular velocity of the electric motor idling is $\omega_{o}=\frac{1500 \cdot \pi}{30}=151.15 \mathrm{rad} \mathrm{s}^{-1}$, the number of revolutions of the electric motor is $n_{o}=1443 \mathrm{rpm}$. Let us accept $n_{o}=$ $1500 \mathrm{rpm}, \omega_{H}=\frac{1500 \cdot \pi}{30}=157 \mathrm{rad} \mathrm{s}^{-1}$. The nominal angular velocity electric motor shaft is $\omega_{H}=145.5 \mathrm{rad} \mathrm{s}^{-1}$. The nominal torque of the electric motor is $M_{H}=A \cdot B \cdot \omega_{H}=$ $11.38 \mathrm{Nm}$. The nominal power of the electric motor is $1.655 \mathrm{~kW}$.

For dynamic analysis of the machine with a slider-crank mechanism let us use the software package SimulationX. Figure 2 shows the dynamic model of the machine with a slider-crank mechanism on SimulationX.

The calculation model was carried out for a typical machine with a slider-crank mechanism and an electric motor of $3 \mathrm{~kW}$ power, and for a synthesized machine with slider-crank mechanism and an electric motor of $1.655 \mathrm{~kW}$ power. Fig. 3 shows a graph of the longitudinal force acting on the slider for a typical machine with a slider-crank mechanism. Fig. 4 shows a graph of the longitudinal force acting on the slider for the synthesized machine with a slider-crank mechanism.

As can be seen from a comparison of Fig. 3 and Fig. 4 the synthesized machine unit with a crankshaft-slide mechanism provides the required technological loading, with less power from the electric motor.

\section{Conclusions}

The method of optimal dynamic synthesis of a machine, which consists of an asynchronous electric motor and a slider-crank mechanism, has been developed. The criterion of optimization of the dynamic synthesis of the machine is built on the basis of the equations of the machine motion with rigid links. The criterion of optimization of the dynamic synthesis of the machine is the root mean square sum of the moments of driving forces, the forces of resistance and inertia forces which are reduced to the axis of rotation of the crank.

The program in Maple for solving this task of the dynamic synthesis of a machine with an asynchronous electric motor and a slider-crank mechanism has been compiled.

The method of optimal dynamic synthesis of a machine, which consists of an asynchronous electric motor and a slider-crank mechanism, has been validated using a numerical simulation of the synthesized machine with a slider-crank mechanism.

In future work, to validate of the new method of optimal dynamic synthesis of a machine, which consists of an asynchronous electric motor and a slider-crank mechanism, we expected to conduct of experimental tests of the synthesized machine.

Edited by: B. Azarhoushang

Reviewed by: two anonymous referees

\section{References}

Astashev, V. K., Babitsky, V. I., and Kolovsky, M. Z.: Dynamics and control of machines, Springer Berlin Heidelberg, 235 pp., 2000.

Browne, J. W.: The Theory of Machine Tools, Cassell and Co. Ltd., 374 pp., 1965.

Dresig, H. and Holzweißig, F.: Dynamics of Machinery, Springer Berlin Heidelberg, 328 pp., 2010.

Dresig, H. and Vulfson, I. I.: Dynamik der Mechanismen, Wien, New York, Springer-Verlag, 621 pp., 1990.

Homer, D. E.: Design of machines and mechanisms, 621 pp., 1998.

Erdman, A. G. and Sandor, G. N.: Mechanism Design: Analysis and Synthesis, Prentice Hall, New Jersey, 688 pp., 1984.

Guangping, H. and Zhiyong, G.: Dynamics synthesis and control for a hopping robot with articulated leg, Mech. Mach. Theory, 46, 1669-1688, 2011. 
Helmi, A. Y. and Hassan, E.-H.: Machining technology: machine tools and operations, Taylor \& Francis Group, LLC, 672 pp., 2008.

Kim, B. S. and Yoo, H. H.: Unified synthesis of a planar four-bar mechanism for function generation using a spring-connected arbitrarily sized block model, Mechanism and Machine Theory, 49, 141-156, 2012.

Kolovsky, M. Z., Evgrafov, A. N., Semenov, Yu. A., and Slousch, A. V.: Advanced theory of mechanisms and machines, Springer Berlin Heidelberg, 396 pp., 2000.

Penunuri, F., Peon-Escalante, R., Villanueva, C., and Pech-Oy, D.: Synthesis of mechanisms for single and hybrid tasks using differential evolution, Mechanism and Machine Theory, 46, 13351349, 2011.

Shabana, A. A.: Dynamic of Multibody Systems, Cambridge University Press, 2nd ed., 384 pp., 1998.
Shchepetil'nikov, V. A.: The balancing of bar mechanisms with unsymmetrical links, Mechanism and Machine Theory, 10, 461466, 1975.

Van der Wijk, V. and Herder, J. L.: Synthesis method for linkages with center of mass at invariant link point - Pantograph based mechanisms, Mech. Mach. Theory, 48, 15-28, 2012.

Vulfson, I. I.: Dynamics of cyclic machines, Springer Berlin Heidelberg. 2015.

Wittenbauer, F.: Graphische Dynamik, Julius Springer, Berlin, 1923.

Wunderlich, W.: Concerning the trajectory of the center of mass of the four-bar linkage and the slider-crank mechanism, J. Mech., 3, 391-396, 1968. 